\title{
A simple microfluidic platform to study age-dependent protein abundance and localization changes in Saccharomyces cerevisiae
}

\author{
Margarita Cabrera ${ }^{1,+}$, Daniele Novarina ${ }^{1}$, Irina L. Rempel ${ }^{1}$, Liesbeth M. Veenhoff ${ }^{1}$, and Michael Chang ${ }^{1, *}$ \\ ${ }^{1}$ European Research Institute for the Biology of Ageing, University of Groningen, University Medical Center Groningen, 9713 AV \\ Groningen, the Netherlands \\ ${ }^{\dagger}$ Present address: Department of Experimental and Health Sciences, Pompeu Fabra University, 08003 Barcelona, Spain \\ * Corresponding Author: \\ Michael Chang, E-mail: m.chang@umcg.nl
}

\begin{abstract}
The budding yeast Saccharomyces cerevisiae divides asymmetrically, with a smaller daughter cell emerging from its larger mother cell. While the daughter lineage is immortal, mother cells age with each cell division and have a finite lifespan. The replicative ageing of the yeast mother cell has been used as a model to study the ageing of mitotically active human cells. Several microfluidic platforms, which use fluid flow to selectively remove daughter cells, have recently been developed that can monitor cell physiology as mother cells age. However, these platforms are not trivial to set up and users often require many hours of training. In this study, we have developed a simple system, which combines a commercially available microfluidic platform (the CellASIC ONIX Microfluidic Platform) and a genetic tool to prevent the proliferation of daughter cells (the Mother Enrichment Program), to monitor protein abundance and localization changes during approximately the first half of the yeast replicative lifespan. We validated our system by observing known age-dependent changes, such as decreased Sir2 abundance, and have identified a protein with a previously unknown age-dependent change in localization.
\end{abstract}

doi: $10.15698 /$ mic2017.05.573

Received originally: 28.03.2017;

In revised form: 06.04.2017,

Accepted 07.04.2017,

Published 13.04.2017.

Keywords: yeast, replicative ageing, microfluidics, CellASIC, protein abundance, protein localization.

\section{Abbreviations:}

MEP - Mother Enrichment Program, $R L S$ - replicative lifespa.

\section{INTRODUCTION}

With the increasing population of elderly people, it is urgent to focus research on ageing and age-associated diseases. In the last few decades, the budding yeast Saccharomyces cerevisiae has been considered a valuable model for understanding longevity in humans $[1,2]$. There are two main models of yeast ageing: replicative and chronological ageing [1, 3]. Replicative lifespan (RLS) refers to the number of daughter cells produced by a mother cell before death. Replicative ageing is thought to be similar to the ageing of asymmetrically dividing cells in higher eukaryotes (e.g. stem cells). Chronological lifespan is the time a cell survives in a non-dividing state, and is used a model for the ageing of non-proliferating cells like our neurons and muscle cells.

Here, we focus on the study of replicatively ageing yeast cells. The classical method to assay RLS is to manually separate a yeast mother cell away from its new-born daughter cells by micromanipulation [4]. A major limitation of this technique is that it is both time and labour intensive. Recently, several microfluidic systems have been developed to allow easier analysis of the RLS of yeast cells [5]. A significant advantage of such systems is the ability to monitor cell physiology and fluorescently tagged proteins during the ageing process on the single-cell level. However, the fabrication and use of these microfluidic platforms requires specific infrastructure and training, which is not readily available in most labs. In this study, we have developed a simple and easy-to-use system that exploits a commercially available microfluidic platform (CellASIC) in combination with the Mother Enrichment Program (MEP) [6], which is a genetic tool to prevent the proliferation of daughter cells, to study changes in protein abundance and localization during replicative ageing in yeast. Our system is designed to monitor these changes early during RLS, when cells are still relatively young, because early changes are more likely to have a causative role in replicative ageing, and most characteristics of the ageing phenotype occur early in the 
a

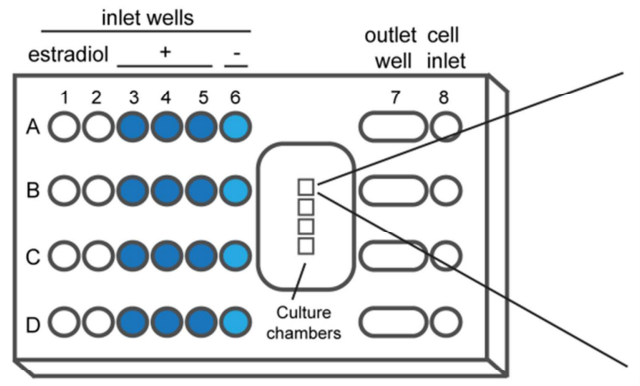

CellASIC plate

b

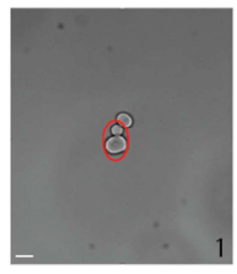

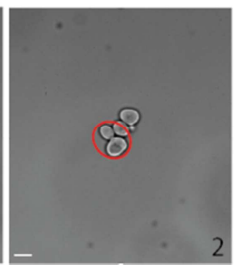

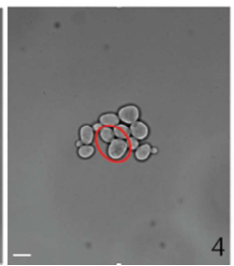

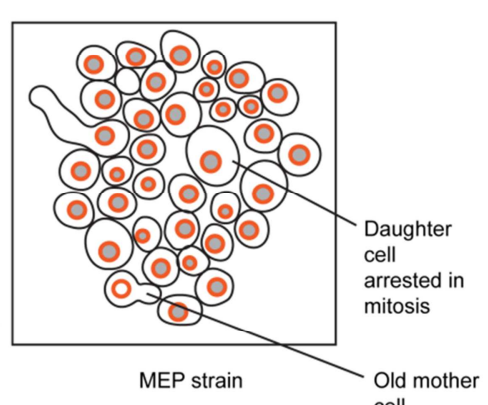

cell
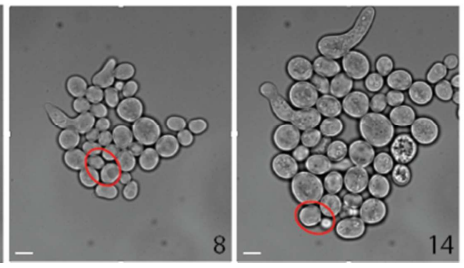

Figure 1: Design of a CellASIC-based platform to study replicative yeast aging. (a) Four strains (A-D) can be monitored for long periods using a CellASIC microfluidic system. Once cells are trapped in the culture chambers, perfusion of the media takes place for $60 \mathrm{~min}$ and perfusion of the media with estradiol is active for $34 \mathrm{~h}$. After addition of estradiol, only the daughter cells of the MEP strain will be arrested in mitosis and cannot proliferate whereas the mother cell continues dividing. Input wells 1 and 2 are not needed for these experiments and are left empty. (b) Bright-field images show the growth of a colony from a founder mother cell (circled in red). Scale bar, $5 \mu \mathrm{m}$.

yeast lifespan [2]. Using our system, we were able to observe several known, and one previously unknown, protein abundance or localization changes that occur during ageing.

\section{RESULTS}

\section{Design of a new system to study yeast ageing}

We have developed a simple platform to monitor changes in protein abundance and localization in individual ageing yeast cells. For this purpose, we used the CellASIC ONIX Microfluidic Platform (Merck Millipore) that allows the analysis of four different strains simultaneously (Fig. 1). Although this device maintains cells growing in a flat monolayer, after several divisions it is challenging to follow a single mother cell due to the rapid growth of surrounding cells descended from the mother cell (Movie S1). To circumvent this limitation, we took advantage of the MEP [6], which restricts proliferation of daughter cells after induction with estradiol (Fig. 1, Movie S2). In the MEP system, a Cre recombinase fused to an estradiol-binding domain (Cre-EBD) is expressed from a daughter-specific promoter; introduction of estradiol causes the Cre-EBD to be transported into the nucleus where Cre disrupts two essential genes, UBC9 and $C D C 20$, in daughter cells [6]. It has previously been demonstrated that most mother cells live a normal lifespan upon activation of the MEP [6]. Consistent with this finding, we found that there is no significant difference in doubling time of wild-type and MEP mother cells grown in the CellASIC device (doubling times: $73 \mathrm{~min}$ and $69 \mathrm{~min}$ for the wild-type and MEP mother cells observed in Movies S1 and S2, respectively).

We designed a strain (MCY699) so that the genetic components of the MEP can be easily combined with a GFP fusion protein of interest from the Yeast GFP Clone Collection [7] by using Synthetic Genetic Array (SGA) methodology [8]. To facilitate analysis of protein localization, we also included Nup49-mCherry as a marker of the nuclear periphery. This strain is available upon request.

\section{Early changes in protein abundance and/or localization} associated with ageing

To validate our microfluidic-based platform, we examined known changes in protein abundance and/or localization that occur as cells age. The NAD-dependent histone deacetylase Sir 2 controls aging by inhibiting recombination at the ribosomal DNA (rDNA) locus [9] and its protein abundance decreases with age [10]. In young cells, we find that Sir2 appears as distinct foci by the nuclear periphery, and the intensity of these foci decreases with age (Fig. 2a).

Protein aggregates associate with Hsp104, forming foci that accumulate in number and intensity with age [11]. In our experiments, Hsp104 localizes to a single nuclear focus in young cells, and as cells age, the focus becomes larger; towards the end of the time-lapse experiment, cytosolic foci can also be seen, and the overall levels of Hsp104-GFP signal increase (Fig. 2b). This result confirms that protein aggregates cannot be cleared and accumulate in aged cells.

Hsp42 is a protein aggregase responsible for the formation of CytoQ deposits where misfolded proteins are retained in the cytosol [12]. Interestingly, an ageassociated protein deposit was recently identified, which is distinct from all known stress-induced protein aggregates; Hsp42 is the first protein localizing to this deposit, promoting its formation [13]. Accordingly, we find that Hsp42 is found in one cytoplasmic focus in young cells that becomes notably larger as cells age (Fig. 2c). This Hsp42-focus is localized mainly in close proximity to the nuclear periphery.

An early change detected with age is the loss of vacuole acidity that has been shown to trigger mitochondria dysfunction [14]. It was reported that mitochondrial membrane potential is reduced in aged cells $[14,15]$, although it 
a
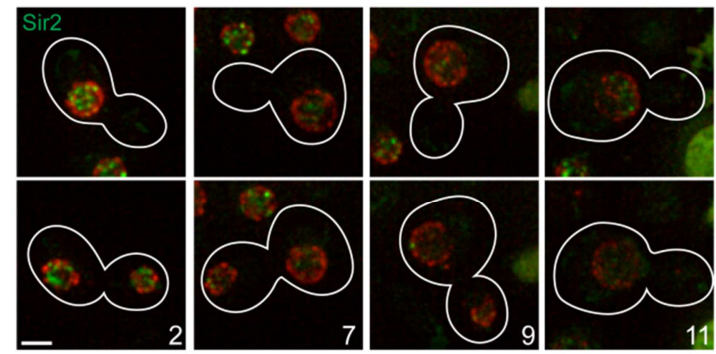

b
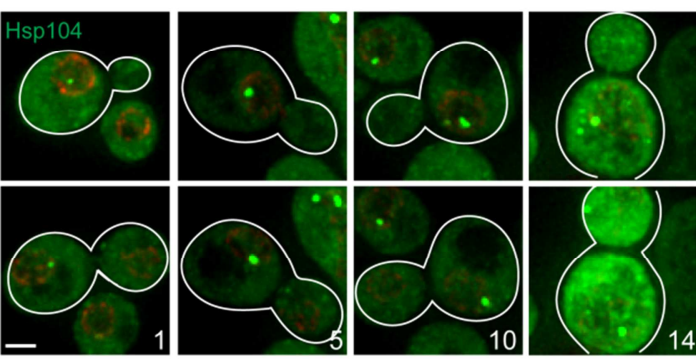

C
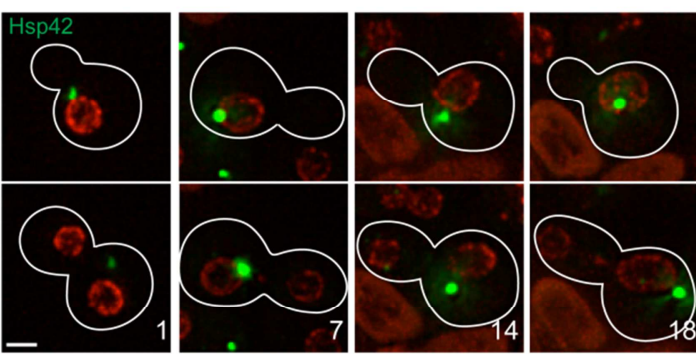

d
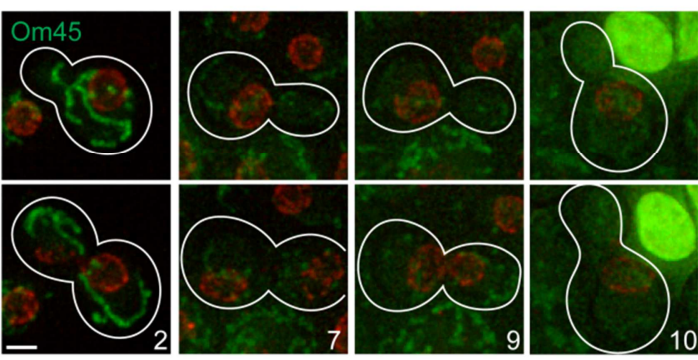

Figure 2: Protein localization changes as cells age. MEP cells expressing Sir2-GFP (a), Hsp104-GFP (b), Hsp42-GFP (c), or Om45GFP (d) were analysed by time-lapse microscopy. Nup49-mCherry was used as marker of the nuclear periphery. Upper and lower images corresponding to the same division, usually 20-60 min apart, are shown to detect possible cell cycle-dependent changes in localization. Stacks of $8-9$ sections $(0.4 \mu \mathrm{m}$ spacing) acquired at different time points were deconvolved (maximal projection is shown). Bright-field images were used to draw cell boundaries and count the number of divisions for the mother cells (age indicated with numbers). Representative images are shown. For Sir2GFP, all six cells analysed showed the same phenotype. For Hsp104-GFP, Hsp42-GFP and Om45-GFP, 10 of 14, 6 of 7 and 11 of 13 cells, respectively, showed the phenotype depicted and described in the text. Scale bar, $2 \mu \mathrm{m}$.

is not clear if this event is common to the whole population [16]. In agreement with previous results [14], we observed that mitochondria labeled with Om45-GFP fragment show a diffuse staining in old cells in contrast to the typical tubular morphology detectable in young cells (Fig. 2d).
These four examples demonstrate the utility of our microfluidic-based platform to study changes in protein abundance and/or localization during replicative aging.

\section{Identification of a novel age-dependent change in protein} localization

We next examined a panel of proteins whose agedependent behaviour is not known. Among these, a previously unidentified age-dependent change was observed in the localization of Pim1, which forms a large focus in aged cells (Fig. 3a). The Lon protease Pim1 is required for the removal of mitochondrial damaged proteins [17], so Pim1 might label a new quality control compartment of the mitochondria. Notably, these enlarged Pim1 foci are not present in daughter cells just after division (Fig. 3b), indicating that they are selectively retained in mother cells. Consistent with a link between Pim1 and ageing, it has been shown that pim1 $1 \Delta$ mutants have a short RLS that can be rescued by overproduction of Hsp104 [18]. A recent study found that Pim1 is important for a new Hsp104-dependent pathway that sequesters and degrades aggregation-prone proteins in the mitochondria [19]. Furthermore, downregulation of Lon promotes cellular senescence in a human melanoma cell line [20].

\section{DISCUSSION}

Microfluidic systems are very powerful tools to examine replicative ageing in yeast [5]. This approach allows the monitoring of ageing cells for many generations, and some of the latest designs make the simultaneous analysis of several strains or conditions possible. Importantly, fluorescent markers can be followed throughout the whole lifespan at the single-cell level. One big disadvantage of existing microfluidic systems is that their use often requires special training and equipment and cannot be easily established in every lab [5]. In contrast, the CellASIC system is commercially available, easy to handle, and can be connected to any inverted microscope. Moreover, daughter cells in this system are not removed by the media flow and can be analysed together with their progenitor cells. In addition, while most microfluidic systems developed so far for the study of yeast replicative ageing are only compatible with haploid cells [5], the CellASIC system is suitable for both haploid and diploid cells, which is relevant because ploidy can impact RLS [21].

A limitation of our platform is that it works best for analysing changes that appear during the first half of the yeast RLS (i.e. within the first 10-15 divisions) because, even with the MEP preventing the proliferation of daughter cells, the mother cell tends to get surrounded by the non-proliferating daughter cells. Thus, our system cannot be used to measure RLS. However, early changes are more likely to have a causative role in the ageing process. Moreover, most age-dependent changes happen early in the yeast lifespan and get progressively worse with age [2, 22]. We were able to reproduce previously published agedependent changes in protein abundance or localization, 
a
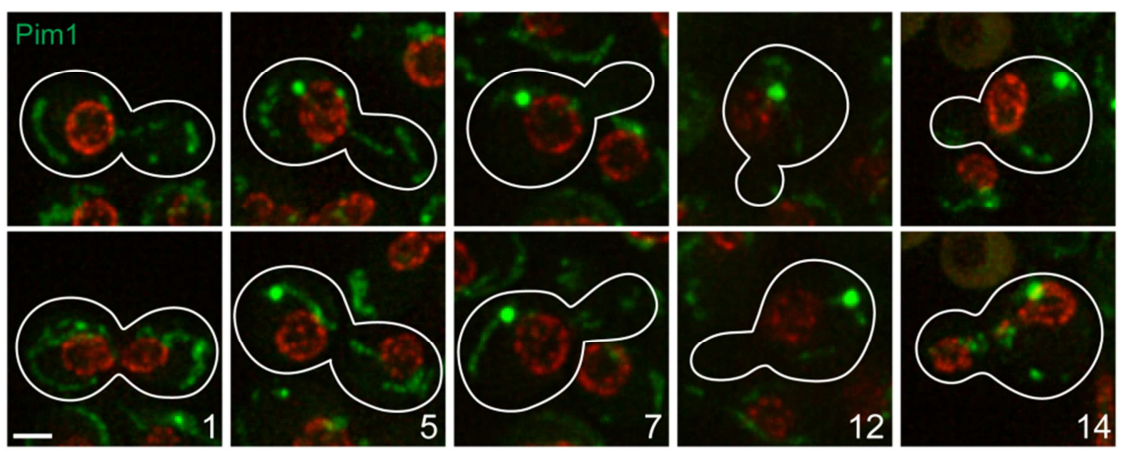

b
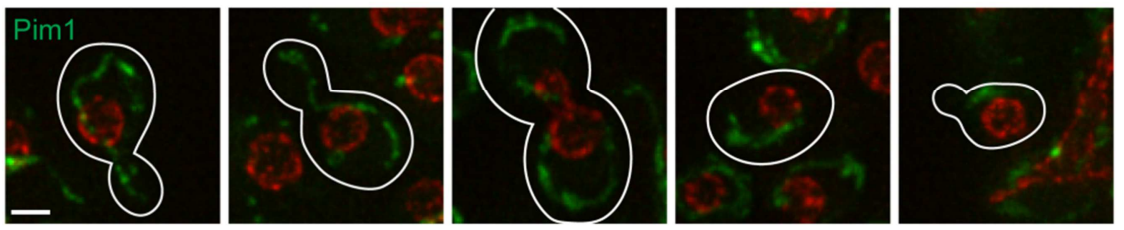

Figure 3: Pim1-GFP localization changes with age. Changes in protein localization were monitored in MEP cells expressing Pim1-GFP (a, b). Nup49-mCherry was used as marker of the nuclear periphery. Upper and lower images corresponding to the same division, but 20-40 min apart, are shown to detect possible cell cycle-dependent changes in localization. Localization of Pim1 in daughter cells (b) is shown. Stacks of 4 sections ( $0.5 \mu \mathrm{m}$ spacing) acquired at different time points were deconvolved (maximal projection is shown). Bright-field images were used to draw cell boundaries and count the number of divisions for the mother cells (age indicated with numbers). Representative images are shown. All five cells analysed showed the same phenotype depicted and described in the text. Scale bar, $2 \mu \mathrm{m}$. thus validating our platform (Fig. 2). We also observed a previously undescribed change in Pim1 localization during replicative ageing (Fig. 3). In aged cells, Pim1 is found in enlarged foci, reminiscent of Tom70 localization, which marks a mitochondrial-derived compartment where the selective degradation of some membrane proteins takes place [16].

A recent tool providing an important contribution to the field of yeast ageing is the MEP [6]. In this study, we added new features to this strain by combining the components of the MEP with the components of the SGA methodology, facilitating the potential high-throughput construction of MEP strains that express protein-GFP fusions from the Yeast GFP Clone Collection. Thus, this system could be scaled up to allow the analysis of agedependent changes in abundance and localization at the proteome-wide level.

\section{MATERIALS AND METHODS}

\section{Yeast strains and molecular biology}

Cells were grown at $30^{\circ} \mathrm{C}$ in synthetic complete (SC) media [23]. The Mother Enrichment Program (MEP) strain (UCC8773 [24]) was provided by Daniel E. Gottschling (Fred Hutchinson Cancer Research Center, Seattle, WA). The replacement of the LEU2 marker with KanMX in the MEP strain was performed using homologous recombination of PCR fragments [25], creating strain MCY662. This strain was then crossed with strain JTY7 [26] (provided by Grant W. Brown, University of Toronto, Canada) by standard yeast genetics to create the strain MCY699. The resulting MEP strain, which also expresses Nup49-mCherry was crossed with the strains from the GFP collection [7] and haploid strains were isolated by sporulation and growth on selective plates.

\section{Microfluidics}

The microfluidic device CellASIC ONIX Microfluidic Platform (Merck Millipore) was used to maintain yeast cells growing in a monolayer for long periods (Fig. 1). A logarithmically growing culture was diluted to an $\mathrm{OD}_{600}$ of 0.1 and $100 \mu \mathrm{l}$ of cells were placed into a microfluidic plate Y04C. Cells were loaded with a pressure of 6 psi for $5 \mathrm{~s}$. SC media was provided first with a pressure of $5 \mathrm{psi}$ for $5 \mathrm{~min}$ to remove the non-trapped cells and then with a pressure of $2 \mathrm{psi}$ for $60 \mathrm{~min}$. Subsequently, SC media containing $1 \mu \mathrm{M}$ estradiol was applied from three different wells with a total pressure of $2 \mathrm{psi}$ (flow rate of $10 \mu \mathrm{l} / \mathrm{h}$ ) for $34 \mathrm{~h}$ (Figure 1). For each time-lapse experiment, we analysed four strains that show similar expression level of the corresponding GFP-proteins and selected 20 individual cells per strain.

\section{Fluorescence microscopy}

Images were acquired using a Deltavision Elite imaging system (Applied Precision (GE), Issaquah, WA, USA) composed of an inverted microscope (IX-71; Olympus) equipped with an PLAPON 60X (1.4 NA) oil immersion objective, InsightSSITM Solid State Illumination, excitation and emission filters for FITC and A594, image-based autofocus, ultimate focus and a coolSNAP HQ2 camera (Photometrics, Tucson, AZ, USA). Timelapse images were acquired with an interval of $20 \mathrm{~min}$ for a total time of $35 \mathrm{~h}$. The exposure time for the FITC channel ranges between 20 and $100 \mathrm{~ms}$. With an exposure time of 100 $\mathrm{ms}$, fluorescent images were acquired every $20 \mathrm{~min}$ in the time-point intervals $1-10$ (i.e. $20 \mathrm{~min}$ time point to $200 \mathrm{~min}$ time point), 42-50, 72-80 and 102-110. A bright-field image acquired at each time point was used to quantify the number of divisions (age). Stacks of 4-9 images with 0.4-0.5 $\mu \mathrm{m}$ spacing were subjected to 3D deconvolution using SoftWoRx 5.5 software (Applied Precision). Processing of all images was performed using Fiji (ImageJ, National Institute of Health).

\section{ACKNOWLEDGEMENTS}

We thank Dan Gottschling and Grant Brown for providing strains, and Fulvio Reggiori and Henning Arlt for sharing the CellASIC system and microscopic expertise. Work in the MC and LMV laboratories was supported by a Vidi grant 
Table 1. Yeast strains used in this study.

\begin{tabular}{|c|c|c|}
\hline Strain name & Relevant genotype & Source \\
\hline UCC8773 & $\begin{array}{l}\text { MATa his } 3 \Delta 1 \text { leu2 } \triangle 0 \text { ura3 } \triangle 0 \text { lys } 2 \Delta 0 \text { ho } \Delta:: P s c w 11 \text {-cre- } \\
\text { EDB78-NatMX loxP-CDC20-intron-loxP-HphMX loxP- } \\
\text { UBC9-IoxP-LEU2 }\end{array}$ & [24] \\
\hline JTY7 & 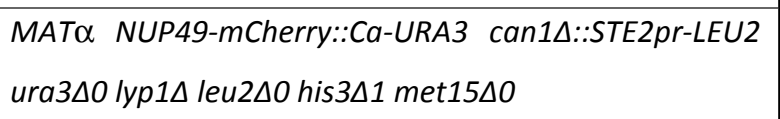 & [26] \\
\hline MCY662 & 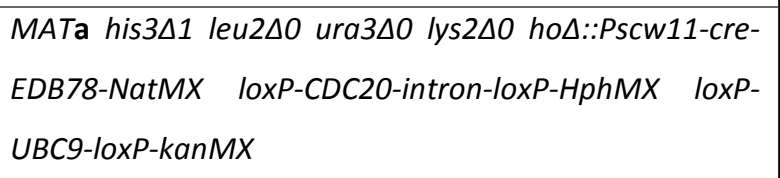 & This study \\
\hline MCY699 & 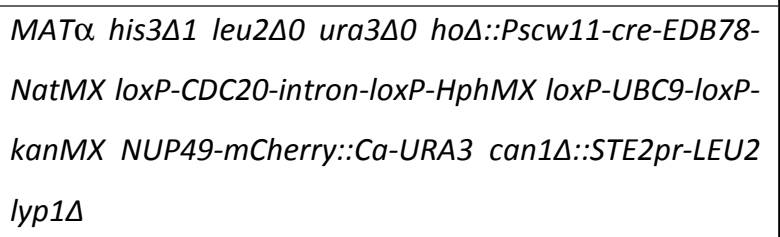 & This study \\
\hline MCSY22 & 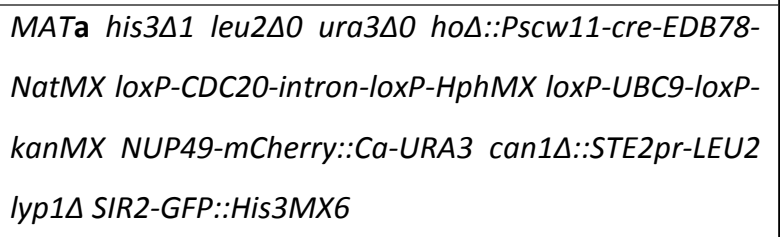 & This study \\
\hline MCSY14 & 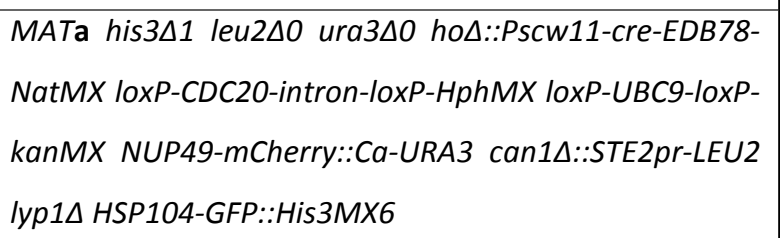 & This study \\
\hline MCSY66 & 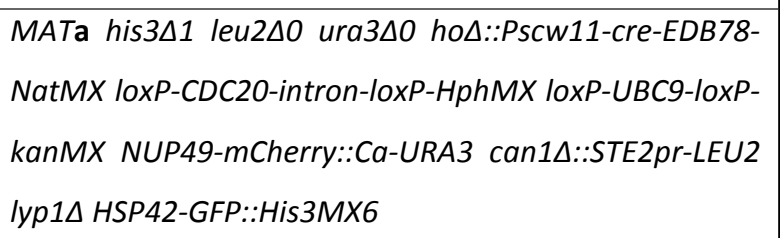 & This study \\
\hline MCSY17 & 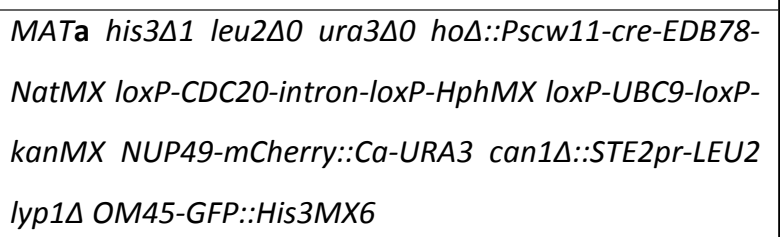 & This study \\
\hline MCSY63 & 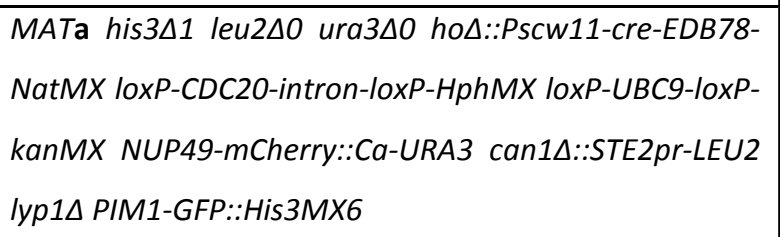 & This study \\
\hline
\end{tabular}


and an ECHO grant, respectively, from the Netherlands Organization for Scientific Research.

\section{SUPPLEMENTAL MATERIAL}

All supplemental data for this article are available online at www.microbialcell.com.

\section{CONFLICT OF INTEREST}

The authors declare no conflict of interest.

\section{REFERENCES}

1. Denoth Lippuner A, Julou T, Barral Y (2014). Budding yeast as a model organism to study the effects of age. FEMS Microbiol Rev 38(2): 300-325.

2. Janssens GE, Veenhoff LM (2016). Evidence for the hallmarks of human aging in replicatively aging yeast. Microbial Cell 3(7): 263-274.

3. Longo VD, Shadel GS, Kaeberlein M, Kennedy B (2012). Replicative and chronological aging in Saccharomyces cerevisiae. Cell Metab 16(1): 18-31.

4. Steffen KK, Kennedy BK, Kaeberlein M (2009). Measuring replicative life span in the budding yeast. J Vis Exp 28.

5. Chen KL, Crane MM, Kaeberlein M (2016). Microfluidic technologies for yeast replicative lifespan studies. Mech Ageing Dev.

6. Lindstrom DL, Gottschling DE (2009). The mother enrichment program: a genetic system for facile replicative life span analysis in Saccharomyces cerevisiae. Genetics 183(2): 413-422.

7. Huh WK, Falvo JV, Gerke LC, Carroll AS, Howson RW, Weissman JS, O'Shea EK (2003). Global analysis of protein localization in budding yeast. Nature 425(6959): 686-691.

8. Tong $A H$, Boone C (2006). Synthetic genetic array analysis in Saccharomyces cerevisiae. Methods Mol Biol 313: 171-192.

9. Kaeberlein M, McVey M, Guarente L (1999). The SIR2/3/4 complex and SIR2 alone promote longevity in Saccharomyces cerevisiae by two different mechanisms. Genes Dev 13(19): 2570-2580.

10. Dang W, Steffen KK, Perry R, Dorsey JA, Johnson FB, Shilatifard A, Kaeberlein M, Kennedy BK, Berger SL (2009). Histone H4 lysine 16 acetylation regulates cellular lifespan. Nature 459(7248): 802-807.

11. Erjavec N, Larsson L, Grantham J, Nystrom T (2007). Accelerated aging and failure to segregate damaged proteins in Sir2 mutants can be suppressed by overproducing the protein aggregation-remodeling factor Hsp104p. Genes Dev 21(19): 2410-2421.

12. Miller SB, Ho CT, Winkler J, Khokhrina $M$, Neuner A, Mohamed MY, Guilbride DL, Richter K, Lisby M, Schiebel E, Mogk A, Bukau B (2015). Compartment-specific aggregases direct distinct nuclear and cytoplasmic aggregate deposition. EMBO J 34(6): 778-797.

13. Saarikangas J, Barral Y (2015). Protein aggregates are associated with replicative aging without compromising protein quality control. Elife 4: e06197.

14. Hughes AL, Gottschling DE (2012). An early age increase in vacuolar $\mathrm{pH}$ limits mitochondrial function and lifespan in yeast. Nature 492(7428): 261-265.

\section{COPYRIGHT}

(C) 2017 Cabrera et al. This is an open-access article released under the terms of the Creative Commons Attribution (CC BY) license, which allows the unrestricted use, distribution, and reproduction in any medium, provided the original author and source are acknowledged.

Please cite this article as: Margarita Cabrera, Daniele Novarina, Irina L. Rempel, Liesbeth M. Veenhoff, and Michael Chang (2017). A simple microfluidic platform to study age-dependent protein abundance and localization changes in Saccharomyces cerevisiae Microbial Cell 4(5): 169-174. doi: 10.15698/mic2017.05.573

15. Veatch JR, McMurray MA, Nelson ZW, Gottschling DE (2009). Mitochondrial dysfunction leads to nuclear genome instability via an iron-sulfur cluster defect. Cell 137(7): 1247-1258.

16. Fehrmann S, Paoletti C, Goulev $Y$, Ungureanu A, Aguilaniu H, Charvin $G$ (2013). Aging yeast cells undergo a sharp entry into senescence unrelated to the loss of mitochondrial membrane potential. Cell Rep 5(6): 1589-1599.

17. Suzuki CK, Suda K, Wang N, Schatz G (1994). Requirement for the yeast gene LON in intramitochondrial proteolysis and maintenance of respiration. Science 264(5156): 273-276.

18. Erjavec N, Bayot A, Gareil M, Camougrand N, Nystrom T, Friguet B, Bulteau AL (2013). Deletion of the mitochondrial Pim1/Lon protease in yeast results in accelerated aging and impairment of the proteasome. Free Radic Biol Med 56: 9-16.

19. Ruan L, Zhou C, Jin E, Kucharavy A, Zhang Y, Wen Z, Florens L, Li R (2017). Cytosolic proteostasis through importing of misfolded proteins into mitochondria. Nature 543(7645): 443-446.

20. Quiros PM, Espanol Y, Acin-Perez R, Rodriguez F, Barcena C, Watanabe K, Calvo E, Loureiro M, Fernandez-Garcia MS, Fueyo A Vazquez J, Enriquez JA, Lopez-Otin C (2014). ATP-dependent Lon protease controls tumor bioenergetics by reprogramming mitochondrial activity. Cell Rep 8(2): 542-556.

21. Kaeberlein M, Kirkland KT, Fields S, Kennedy BK (2005). Genes determining yeast replicative life span in a long-lived genetic background. Mech Ageing Dev 126(4): 491-504.

22. Janssens GE, Meinema AC, Gonzalez J, Wolters JC, Schmidt A, Guryev V, Bischoff R, Wit EC, Veenhoff LM, Heinemann M (2015). Protein biogenesis machinery is a driver of replicative aging in yeast. elife 4: e08527.

23. Treco DA, Lundblad V (2001). Preparation of yeast media. Curr Protoc Mol Biol Chapter 13: Unit 13.1

24. Henderson KA, Hughes AL, Gottschling DE (2014). Motherdaughter asymmetry of $\mathrm{pH}$ underlies aging and rejuvenation in yeast. Elife 3: e03504

25. Janke C, Magiera MM, Rathfelder N, Taxis C, Reber S, Maekawa $H$, Moreno-Borchart A, Doenges G, Schwob E, Schiebel E, Knop M (2004) $A$ versatile toolbox for PCR-based tagging of yeast genes: new fluorescent proteins, more markers and promoter substitution cassettes. Yeast 21(11): 947-962.

26. Tkach JM, Yimit A, Lee AY, Riffle M, Costanzo M, Jaschob D, Hendry JA, Ou J, Moffat J, Boone C, Davis TN, Nislow C, Brown GW (2012). Dissecting DNA damage response pathways by analysing protein localization and abundance changes during DNA replication stress. Nat Cell Biol 14(9): 966-976. 